1 Does $\boldsymbol{G}_{\boldsymbol{S T}}$ underestimate genetic differentiation from

2

3

4

5

6

7 Institute of Zoology, Zoological Society of London, London NW1 4RY, 8 United Kingdom

3

4

5

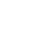

J. Wang

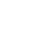

\title{
marker data?
} 9 
10

11 Right running head: Correlation of $G_{S T}$ and $H_{S}$

12 Key words: Population Structure, Genetic Differentiation, $F_{S T}, G_{S T}$, Markers, Drift, Mutations

13 Corresponding author

14

15

16

17

18

19

20

21

22

23
Jinliang Wang

Institute of Zoology

Regent's Park

London NW1 4RY

United Kingdom

Tel: 00442074496620

Fax: 00442075862870

Email: jinliang.wang@ioz.ac.uk 


\begin{abstract}
The widely applied genetic differentiation statistics $F_{S T}$ and $G_{S T}$ have recently been criticised for underestimating differentiation when applied to highly polymorphic markers such as microsatellites. New statistics claimed to be unaffected by marker polymorphisms have been proposed and advocated to replace the traditional $F_{S T}$ and $G_{S T}$. This study shows that $G_{S T}$ gives accurate estimates and underestimates of differentiation when demographic factors are more and less important than mutations, respectively. In the former case, all markers, regardless of diversity $\left(H_{S}\right)$, have the same $G_{S T}$ value in expectation and thus give replicated estimates of differentiation. In the latter case, markers of higher $H_{S}$ have lower $G_{S T}$ values, resulting in a negative, roughly linear correlation between $G_{S T}$ and $H_{S}$ across loci. I propose that the correlation coefficient between $G_{S T}$ and $H_{S}$ across loci, $r_{G H}$, can be used to distinguish the two cases and to detect mutational effects on $G_{S T}$. A highly negative and significant $r_{G H}$, when coupled with highly variable $G_{S T}$ values among loci, would reveal that marker $G_{S T}$ values are affected substantially by mutations and marker diversity, underestimate population differentiation, and are not comparable among studies, species and markers. Simulated and empirical datasets are used to check the power and statistical behaviour, and to demonstrate the usefulness of the correlation analysis.
\end{abstract}

\title{
Introduction
}

A species rarely breeds at random throughout its whole range to form a homogenous unit. Frequently a species is genetically structured in space, subdivided into subunits called demes, races, subpopulations, ... Delineating the spatial genetic structure by dividing a species into subunits and quantifying the genetic differentiation among the subunits is important in many biological fields such as evolution, conservation, human medicine and forensics. The subdivision can be made based on natural (e.g. rivers) or artificial (e.g. dams or highways) boundaries, on geographical locations, or on genetic data (e.g. Pritchard et al. 2000), and the differentiation can be measured from marker data using Wright's (1943) $F_{S T}$, Nei's (1973) $G_{S T}$ and related statistics such as Weir \& Cockerham's (1984) $\theta$ and Slatkin's (1995) $R_{S T}$. The development and wide application of highly polymorphic markers such as microsatellites made these statistics ever more popular, but also caused some confusion and concern. The most popular differentiation statistics, $F_{S T}$ and $G_{S T}$, are believed to underestimate population differentiation when calculated from markers of high diversity (e.g. Nagylaki 1998; Hedrick 
2005; Jost 2008), and for this reason alternative statistics were proposed and advocated to replace them (Hedrick 2005; Jost 2008; Meirmans \& Hedrick 2011). The new differentiation statistics, however, are criticized for their lack of biological meaning and applications, their marker dependency but drift independency, and so on (see Ryman \& Leimar 2009; Whitlock 2011; Wang 2012).

The claim that $F_{S T}$ and $G_{S T}$ underestimate population differentiation is made from both theoretical and empirical grounds. The mathematical definition of $G_{S T}=\left(H_{T}-H_{S}\right) / H_{T}$ suggests that it cannot take values larger than the average within subpopulation homozygosity, $1-H_{S}$ (Jin \& Chakraborty 1995; Nagylaki 1998; Hedrick 1999, 2005). This constraint is true both mathematically and biologically. Both $F_{S T}$ and $G_{S T}$ are inherently constrained by $H_{S}$, as they signify the amount of genetic variation between populations $\left(V_{B}\right)$ as a proportion of the total variation $V_{T}$, which is composed of within $\left(V_{W}\right)$ and between $\left(V_{B}\right)$ population variation. A high $H_{S}$ means a high $V_{W}$, and necessarily a low $V_{B}$ as a proportion of $V_{T}$ (i.e. low $F_{S T}$ and $\left.G_{S T}\right)$. However, the constraint imposed on $F_{S T}$ and $G_{S T}$ by $H_{S}$ does not necessarily mean they are always marker $H_{S}$ dependent and underestimate differentiation from markers of high $H_{S}$, as claimed by some authors (e.g. Nagylaki 1998; Hedrick 1999, 2005; Jost 2008). On the empirical grounds, some studies showed that $G_{S T}$ based on highly polymorphic microsatellites is usually lower than $G_{S T}$ based on weakly polymorphic allozyme loci (e.g. Sanetra \& Crozier 2003), and is obviously too low for highly differentiated subspecies (e.g. Balloux et al. 2000; Carreras-Carbonell et al. 2006). These empirical evidences are true for these particular systems, but do not suggest that $F_{S T}$ and $G_{S T}$ calculated from highly polymorphic markers must always underestimate population differentiation in all circumstances.

Are $F_{S T}$ and $G_{S T}$ dependent on marker diversity? Do they always underestimate population differentiation from markers of high diversity (e.g. microsatellites)? Under which set of conditions do they provide marker dependent (and thus biased) and marker independent (and thus accurate) estimates of population differentiation? Is it possible to detect whether $F_{S T}$ and $G_{S T}$ values calculated from a set of markers underestimate differentiation or not? In this paper, I will use a combination of analytical modelling, simulated data and empirical data to answer these questions. I show $G_{S T}$ is independent of $H_{S}$ when mutation rate $(u)$ is small relative to migration rate $(m)$ or drift $(1 / 2 N)$. Otherwise, $G_{S T}$ decreases nearly linearly with an increase in $H_{S}$. The results suggest a test for the presence or absence of mutational effects on $G_{S T}$. If single-locus $G_{S T}$ values are highly variable and the correlation between single-locus 
$G_{S T}$ and $H_{S}$ values is significantly negative, then the observed $G_{S T}$ values are substantially affected by mutations, are locus specific, and seriously underestimate the differentiation due to population demography. If the correlation is insignificant, then the observed single-locus $G_{S T}$ values are unaffected by mutations and are marker independent. They can then be averaged to give an overall estimate of the genetic differentiation caused by demography only. Simulations and empirical data are analysed to check the power and statistical properties of the correlation and regression analyses.

\section{Method}

The relationship between $G_{S T}$ and $H_{S}$ is investigated by analyses of standard population genetics models of migration, drift and mutation. The results are then verified by analyses of simulated and empirical datasets.

\section{Theory}

Following most previous studies of $F_{S T}$, I assume a population under the finite island model of migration (Wright 1931) and the infinite allele model of mutation (Kimura \& Crow 1964) for mathematical tractability. The results and conclusions are, however, applicable qualitatively to populations under other migration models, such as Wright's (1943) isolation by distance or neighbourhood model and Kimura \& Weiss's (1964) stepping stone model, and under other mutation models, such as stepwise mutation model for microsatellites or allozymes (Ohta \& Kimura 1973).

Under the finite island model with migration rate $m$ among $s$ subpopulations of effective size $N$, and under the infinite allele model for a neutral locus with mutation rate $u$, the recurrence equations for the expected homozygosity within a subpopulation, $J_{0}$, and between two subpopulations, $J_{1}$, is (Nei 1975; Li 1976)

$J_{0(t+1)}=d\left(a\left(c+(1-c) J_{0(t)}\right)+(1-a) J_{1(t)}\right)$

$J_{1(t+1)}=d\left(b\left(c+(1-c) J_{0(t)}\right)+(1-b) J_{1(t)}\right)$

where $b=m(2-m) / s, a=(1-m)^{2}+b, c=1 /(2 N)$ and $d=(1-u)^{2}$. Equivalently, $J_{0}$ and $J_{1}$ are the probabilities that two genes taken at random from within a subpopulation and from different subpopulations, respectively, are identical in state. The complements, $H_{S}$ $=1-J_{0}$ and $H_{1}=1-J_{1}$, give the expected (i.e. assuming random union of gametes) 
heterozygosity or gene diversity (Nei 1973) within and between subpopulations. The total expected heterozygosity or gene diversity in the entire population is $H_{T}=\left(H_{S}+(s-\right.$ 1) $\left.H_{1}\right) / s=1-J_{1}-\left(J_{0}-J_{1}\right) / s\left(\right.$ Nei 1975). Given $H_{T}$ and $H_{S}, G_{S T}$ is calculated by $G_{S T}=$ $1-H_{S} / H_{T}$ (Nei 1973). Using (1) and (2), we can calculate recurrently the values of $H_{S}, H_{T}$ and $G_{S T}$ at each generation, given parameters $m, N, u, s$ and initial gene identities $J_{0(0)}$ and $J_{1(0)}$.

Under the joint action of mutation, migration and drift at rates $u, m$, and $1 /(2 N)$ respectively, the gene diversity $\left(H_{S}, H_{T}\right)$ and its distribution $\left(G_{S T}\right)$ will reach equilibrium values. $G_{S T}$ attains its equilibrium value much faster than $H_{S}$ and $H_{T}$, because it is determined by the strongest (in terms of rate) while $H_{S}$ and $H_{T}$ are determined by the weakest among the forces of mutation, migration and drift. The equilibrium gene identity values are (Nei 1975; Li 1976)

$J_{0(\propto)}=c d(a-(a-b) d) / G$

$J_{1(\propto)}=c d b / G$

where $G=1-d(a(1-c)+1-b)+d^{2}(a-b)(1-c)$. The equilibrium gene diversity and differentiation values, $H_{S(\propto)}, H_{T(\propto)}$ and $G_{S T(\propto)}$, can be calculated using (3) and (4). The expression for $G_{S T(\propto)}$ is complicated, but can be simplified approximately to (Takahata \& Nei 1984)

$G_{S T(\propto)} \approx 1 /\left[1+2 N\left(\frac{s}{s-1}\right)\left(\frac{1}{(1-m)^{2}(1-u)^{2}}-1\right)\right]$.

When $m, u \ll 1,(5)$ is further simplified to (Takahata \& Nei 1984)

$G_{S T(\propto)} \approx 1 /\left[1+4 N\left(\frac{s}{s-1}\right)(m+u)\right]$

When $s \rightarrow \propto$, (6) again reduces to the equilibrium $F_{S T}$ of the infinite island model of Wright (1969, page 291), indicating that $F_{S T}$ and $G_{S T}$ are equivalent (Nei 1977; Takahata \& Nei 1984).

Although several studies have used similar models to investigate the impact of mutations on $F_{S T}$ and $G_{S T}$ (e.g. Ryman \& Leimar 2008; Whitlock 2011), none has examined the direct relationship between $G_{S T}$ and $H_{S}$. Herein I will use equations (1-6) to explore this relationship in populations in both equilibrium and non-equilibrium conditions under 
different parameter $(m, u, N, s)$ combinations. This is important as both $G_{S T}$ and $H_{S}$ are estimable from marker data, and examining the observed patterns of $G_{S T}$ and $H_{S}$ at a set of marker loci sheds light on the possible impact of mutations on $G_{S T}$.

\section{Simulations}

Simulated data typical of those encountered in practice were generated to test whether the correlation analysis of single locus estimates of $G_{S T}$ and $H_{S}$ could be used to detect the effect of mutations on $G_{S T}$ when it is present, and whether the analysis does not falsely detect the effect of mutations when it is absent. The behaviour and power of the correlation analysis were investigated by analysing simulated data with varying sampling intensities (of individuals from a subpopulation, of subpopulations, and of markers), different population properties $(N, s, m, u)$ and different mutation and migration models.

The simulations considered the finite island model as described above, and a onedimensional circular stepping stone model (Kimura \& Weiss 1964). In the latter model, a number of $s$ subpopulations are arranged in a circle and each subpopulation receives a proportion $m / 2$ of its individuals from each of its two neighbouring subpopulations. In both models, each subpopulation is composed of $N$ diploid monoecious individuals. At each discrete generation, the events are mutations, migrations and reproductions occurring in that order. Mutations are assumed to follow either the infinite allele model or the stepwise mutation model. For the former, a mutation always generates a novel allele the population has never seen before. For the latter, the mutated allele increases or decreases in size by 1 repeat with an equal probability of 0.5 . For both models, the number of new mutations at a locus in each subpopulation at each generation was sampled from a Poisson distribution with parameter value $2 \mathrm{Nu}$. For each new mutation, a gene was drawn at random from the $2 \mathrm{~N}$ genes and was changed according to the mutation model. Reproduction is assumed to be random union of gametes, such that selfing and outbreeding occur at rates $1 / N$ and $1-1 / N$ respectively, and the effective size is equal to the census size for each subpopulation.

An ancestral population was assumed to be the same as the subdivided population described above except for population size and structure. It was unsubdivided and had a size $N_{A}=r s N$, where $r=0.5,1$ and 2 such that it had equilibrium genetic diversity smaller than, close to, and larger than the subdivided population respectively. The ancestral population was maintained for a large number of generations for it to reach mutation-drift equilibrium at a neutral locus with mutation rate $u$ (which was variable among a number of $L$ loci). It was then 
subdivided into $s$ subpopulations of size $N$, which were maintained as described above for $g$ $(=100,200,400)$ generations or for a sufficiently large number of generations, in the order of $\operatorname{Max}(1 / u, 1 / m, 2 N)$, to reach mutation-drift-migration equilibrium. A sample of $M$ individuals was then taken at random from each of $R(\leq s)$ randomly selected subpopulations, and each sampled individual was genotyped at a number of $L$ loci.

The genotype data were then used to calculate Nei \& Chesser's (1983) nearly unbiased estimators of $H_{S}, H_{T}$, and thus $G_{S T}$,

$\widehat{H}_{S}=\frac{2 \widetilde{M}}{(2 \widetilde{M}-1) R} \sum_{j=1}^{R}\left(1-\sum_{i=1}^{k} x_{i j}^{2}\right)$,

$\widehat{H}_{T}=1-\sum_{i=1}^{k}\left(\frac{1}{R} \sum_{j=1}^{R} x_{i j}\right)^{2}+\frac{\widehat{H}_{S}}{2 \widetilde{M} R}$

$\widehat{G}_{S T}=1-\widehat{H}_{S} / \widehat{H}_{T}$,

where $x_{i j}$ is the frequency of allele $i$ in the sample from subpopulation $j, k$ is the number of alleles observed in the set of samples from the $R$ subpopulations, and $\widetilde{M}$ is the harmonic mean sample sizes ( $\equiv M$ in the simulations).

The estimates $\widehat{H}_{S}$ and $\widehat{G}_{S T}$ were then used to calculate their correlation coefficient $r_{G H}$ across loci. The significance of $r_{G H}$ was tested by a permutation analysis in which $\widehat{H}_{S}$ and $\widehat{G}_{S T}$ were both randomized across loci before calculating $r_{G H}$ in $10^{6}$ replicates. The proportion of replicates in which $r_{G H}$ was smaller than the $r_{G H}$ value calculated from the original data was taken as the $p$ value. The correlation coefficient was taken as statistically significant when $p<0.001$. A significant negative correlation $r_{G H}$ indicates that $\widehat{G}_{S T}$ has been affected by mutations and thus underestimates the differentiation caused purely by demography (drift and migration). Otherwise, markers with different levels of diversity $\widehat{H}_{S}$ are equally differentiated, they all give the same $G_{S T}$ expected from the impact of drift and migration only, and the single locus $G_{S T}$ estimates can be averaged to give a better (in precision) overall estimate of differentiation.

Too many parameter combinations, due to the numerous parameters and the numerous plausible values of each parameter, are involved in determining $\widehat{H}_{S}$ and $\widehat{G}_{S T}$ that a realistic simulation study can only consider a small fraction of them. I studied the effect of each parameter in isolation of others each time by varying the values of the focal parameter only (see Table 1). For each parameter combination, a number of 100 replicate datasets were 
generated and analysed. The analysis results were reported as the mean correlation coefficient between $\widehat{G}_{S T}$ and $\widehat{H}_{S}, \bar{r}_{G H}$, and the proportion of replicates with a statistical significant (at $p<0.001) r_{G H}$ among the 100 replicates.

\section{The simulation program was checked by comparing the simulated against the} predicted values of several quantities to make sure it worked properly. First, the effective size of the entire population in the finite island model is $N_{e}=s N /\left(1-F_{S T}\right)$ (Wright 1943; Wang \& Caballero 1999), where $F_{S T}$ can be replaced by $G_{S T}$. This theoretical prediction was compared with that estimated from the simulated pedigrees, using the formula $\frac{1}{2 N_{e}}=\frac{\theta_{t+1}-\theta_{t}}{1-\theta_{t}}$ where $t$ the generation is large and $\theta_{t}$ is the average coancestry at generation $t$ for all individuals in the entire population. Second, the predicted values of $H_{S}, H_{T}$ and $G_{S T}$ by (3-4) were compared with the corresponding observed values for an equilibrium population under infinite allele and finite island models. In all situations investigated, the predicted and estimated (observed) values fitted very well.

\section{Empirical data}

The simulation model may be too simple to reflect the reality. In a real population, both $m$ and $N$ may vary over space and time, and migrations and mutations may not follow the ideal models assumed in the simulations. Supplementing simulations, therefore, I also analysed several recently published empirical datasets to demonstrate the use of the proposed correlation analysis.

Atlantic Salmon: To investigate the genetic structure of Atlantic salmon populations in the entire North American range of the species, Moore et al. (2014) sampled 9142 individuals from 153 populations and genotyped each individual at 15 microsatellite loci. They also sampled 1080 individuals from 50 populations and genotyped each individual at 3192 SNP loci. The two datasets were analysed separately in the present study of the relationship between $G_{S T}$ and $H_{S}$.

Blacknose sharks: Using 23 microsatellites and mtDNA sequences, Portnoy et al. (2014) investigated the genetic structure and barriers to gene flow of 10 blacknose shark populations sampled (651 individuals in total) from the western North Atlantic Ocean. It was found that the $F_{S T}$ values at the 23 microsatellite loci between the Bahamas and any of the other populations were more than an order of magnitude greater than the values between any two of the other populations. Therefore, $G_{S T}$ and $H_{S}$ values were calculated for each locus in the 2 
alternative population structures, the 10- and 2-population (Bahamas and the rest) models in the present study.

Mediterranean shore crab: Schiavina et al. (2014) investigated the genetic structure of the Mediterranean shore crab (Carcinus aestuarii) in the Adriatic Sea (central Mediterranean), using 11 polymorphic microsatellites in 431 individuals collected from eight sites. One locus, Cae30, has only 5 alleles and a gene diversity of $H_{S}=0.1$, much lower than the locus with the 2nd lowest diversity, which has 13 alleles and a $H_{S}=0.77$. So Cae30 was excluded as an obvious outlier from the $G_{S T}$ and $H_{S}$ correlation analysis.

Blacktip reef sharks: To understand the genetic structure of blacktip reef sharks

(Carcharhinus melanopterus), Vignaud et al. (2014) sampled 758 individuals from 15 sites (4 widely separated locations in the Indo-Pacific and 11 islands in French Polynesia) widely distributed in the Indian and Pacific Oceans. Each sampled individual was genotyped at 17 microsatellite loci. Three loci (cil169, cli107 and cli12) were found to deviate significantly from Hardy-Weinberg equilibrium and were suspected to contain null alleles (Vignaud et al. 2014). The three loci were excluded from their original genetic analysis. Herein I investigated the impact of mutations on the estimated differentiation among these shark populations by analysing the relationship between $G_{S T}$ and $H_{S}$, using both the entire set of 17 loci and the selected subset of 14 loci.

Copper rockfish: Using 17 microsatellite DNA loci, Dick et al. (2014) assessed the genetic diversity of and the differentiation among ten populations of copper rockfish (Sebastes caurinus) representing paired samples of outer coast and the heads of inlets in five replicate sounds on the west coast of Vancouver Island, British Columbia. The sample size per population varies between 30 and 105 . I calculated the $G_{S T}$ and $H_{S}$ values at each of the 17 loci among the 10 populations, and tested whether the marker differentiation is affected by mutations or not.

\section{Results}

\section{Analytical results}

Equation (6) suggests that $G_{S T}$ at neutral loci is determined by the joint action of migration, mutation and drift occurring at rates $m, u$, and $1 /(2 N)$ respectively. The relative impact of each evolutionary force on $G_{S T}$ is determined by its rate as a proportion of the total rate, $m+u+1 /(2 N)$. When subpopulations are small such that drift is the dominating force (i.e. 
$1 /(2 N) \gg u+m)$, then $H_{S} \rightarrow 0$ (i.e. fixation) and $G_{S T(\propto)} \rightarrow 1$ in equilibrium conditions. When mutation is weak relative to drift and migration (i.e. $u \ll 1 /(2 N)+m$ ), then $G_{S T(\propto)} \approx$ $1 /\left[1+4 N\left(\frac{s}{s-1}\right) m\right]$, which suggests that $G_{S T(\propto)}$ reflects demography only and all loci with varying but small $u$ have the same expected $G_{S T}$. In contrast, for loci with a high $u$ in a population with a large $N$ and a small $m$ (i.e. $u » 1 /(2 N)+m$ ), $G_{S T(\propto)}$ becomes locus (or mutation) dependent and covaries with locus specific $H_{S}$ (below). In such a case, marker based $G_{S T(\propto)}$ has little bearing on population demography, the $G_{S T(\propto)}$ value calculated from one set of loci can hardly be congruent with that from another set of loci, and it is incomparable among studies, species and loci.

Figure 1 plots the equilibrium $G_{S T}$ as a function of $H_{S}$, calculated by (5) and (3) respectively, for different parameter combinations of $u, m$ and $N$, assuming $s=10$. When differentiation is expected to be small due to either strong migration $(m \geq 0.01)$ or weak drift ( $N \geq 2500), G_{S T}$ keeps constant and does not vary with $H_{S}$ in its entire range of $[0,1]$ caused by widely varying $u$ values in range of $\left[10^{-6}, 10^{-2}\right]$. The observation disproves the belief that $G_{S T}$ underestimates differentiation and becomes $H_{S}$ dependent when $H_{S}$ is high (e.g. Nagylaki 1998; Hedrick 1999, 2005; Jost 2008). High $H_{S}$ values (say 0.95) do constrain $G_{S T}$ to small values with a maximum of $1-H_{S}$, but do not necessarily lead to underestimated and locusvarying $G_{S T}$. What is relevant is the main mechanism (determined by the relative strengths of mutation, drift and migration) leading to the observed high $H_{S}$, not the observed high $H_{S}$ per $s e$. A high $H_{S}$ is usually due to a high $u$ or/and a high $N$. However, as long as $m$ is much higher than $u, G_{S T}$ is virtually independent of $H_{S}$.

When drift is strong (i.e. $N$ small) and migration is weak relative to mutations, $G_{S T}$ decreases almost linearly with an increasing $H_{S}$ due to an increasing $u$ (Figure 1). Only in this situation is the belief that $G_{S T}$ covaries with $H_{S}$ (e.g. Nagylaki 1998; Hedrick 1999, 2005; Jost 2008 ) certified. For the parameter combination $N=250, m=0.001$, and $s=10$ in Figure 1 , for example, $G_{S T}$ keeps almost a constant value of 0.45 when $u$ varies between $10^{-6}$ and $3 \times 10^{-6}$ that leads to a $H_{S}$ varying between 0 and 0.5 . With $u>3 \times 10^{-6}$ and thus $H_{S}>0.5, G_{S T}$ begins to decrease linearly with an increasing $H_{S}$ (or $u$ ). Similar results are obtained with other values of the number of subpopulations $(s)$.

Many generations, in the order of $1 / m, 1 / u$ or $2 N$ whichever is the smallest, are required for a subdivided population to reach the equilibrium differentiation. Natural 
populations may never reach such equilibrium as $m$ and $N$ are constantly changing. It is thus important to check whether the above observations (Figure 1) also apply to non-equilibrium populations. Figure 2 plots $G_{S T}$ as a function of $H_{S}$ at generations 50, 200 and 1000 since the subdivision. Mutation rate $(u)$ is assumed to vary from $10^{-6}$ to $10^{-2}$, and the initial gene diversity is assumed to be $J_{0(0)}=J_{1(0)}$ and to take values $r J_{0(\propto)}$, where $r=1,0.5$ and 0.25 . The relationship between $G_{S T}$ and $H_{S}$ in a non-equilibrium population is similar to that in an equilibrium population (Figure 1 ). Whenever $u \ll 1 /(2 N)+m, G_{S T}$ does not vary with $H_{S}$ (or $u$ ). Depending on $u$ as well as $N$ and $m, H_{S}$ can freely vary in almost the entire range of $[0,1]$ without affecting the value of $G_{S T}$. Otherwise, $G_{S T}$ decreases nearly linearly with an increasing $H_{S}$ ( or $u$ ). The further away a population departs from the equilibrium, the less affected it is by mutations because the latter require time to accumulate. When $N=250$, for example, mutations start to have a substantial impact on $G_{S T}$ at generations 50, 200 and 1000 when $H_{S} \geq 0.8(u \geq 0.0015), H_{S} \geq 0.5(u \geq 0.00001)$ and $H_{S} \geq 0.3(u \geq 0.00003)$ respectively. Initial gene identities (or diversities) seem to have little effect on the relationship between $G_{S T}$ and $H_{S}$ at any generation.

\section{Simulation results}

Confirming the analytical results presented above, simulations show that, when mutations are strong relative to migrations $(m=0.001), G_{S T}$ estimates vary among loci that have different $u$ and thus different $H_{S}$, and are negatively correlated with $H_{S}$ (Figure 3 ). This is true for the finite island and stepping stone migration models, and for the infinite allele, finite allele and stepwise mutation models. This is also true no matter the population is at mutation-driftmigration equilibrium (Figure 3) or not (data not shown). The negative correlation in stepping stone migration model and infinite allele mutation model is stronger than that in other migration and mutation models. In contrast, when mutations are weak relative to migrations ( $m=0.01), G_{S T}$ estimates are small and are almost constant among loci with different $u$ and thus different $H_{S}$. This is shown for an equilibrium population under different migration and mutation models (Figure 3), but is also true for non-equilibrium populations (data not shown).

When migrations are weak relative to mutations such that $G_{S T}$ is substantially affected by $u$ and becomes negatively correlated with $H_{S}$, a modest sampling effort is needed to detect the correlation for different migration and mutation models (Figure 4). This is also true for populations that have not reached mutation-drift-migration equilibrium (data not shown). Setting the statistical significance at a conservative level of $p<0.001$, the false detection rate 
of mutational effects is low (generally below 7\%), while the power is generally above $60 \%$ except when less than 10 loci and less than 4 subpopulations are used in the analysis. In agreement with the results in Figure 3, the correlation analysis is less powerful for the island migration model coupled with the stepwise or 2-allele mutation model than other models. While the power increases with the numbers of sampled loci and sampled subpopulations (Figure 4), it is little affected by the number of sampled individuals per subpopulation, $M$, as long as $M>10$. This is not surprising because the population is highly differentiated for the parameter combinations and just a few individuals per subpopulation would allow for a good estimate of $G_{S T}$.

\section{Empirical analysis}

The Atlantic salmon data clearly show an extremely strong negative correlation $(r=-0.953)$ between $G_{S T}$ and $H_{S}$ estimates among the 15 microsatellites (Figure 5A), with a $p$ value of $0.0 \times 10^{-6}$. These markers are highly polymorphic, with $H_{S}$ varying between 0.66 and 0.94 and with the number of observed alleles varying between 15 and 91. Compatible with a substantial impact of mutations, these markers have low but highly variable $G_{S T}$ values, varying between 0.02 and 0.09 with a mean of 0.045 and a coefficient of variation of 0.629 . These single locus $G_{S T}$ values are all highly significant, as determined by permutation (permuting individuals among subpopulations) tests.

In contrast, the correlation between $G_{S T}$ and $H_{S}$ estimates of the 3192 SNPs (Figure 5B) is positive and small ( $r=0.044)$, with a $p$ value of 0.993 which is insignificant. $H_{S}$ values distribute nearly uniformly in the range $[0,0.5]$. While most SNPs have $G_{S T}$ values of about 0.1, quite a few outliers show $G_{S T}$ values well above 0.4. The mean $G_{S T}$ is 0.099 for the 3192 SNPs and is 0.091 when the outlier SNPs with $G_{S T}>0.3$ are removed. Both values are much larger than the mean $G_{S T}$ across the 15 microsatellites which is 0.045 . The comparison between SNPs and microsatellites further verifies that the differentiation at microsatellites is greatly impacted by mutations and thus underestimates the underlying population differentiation due to demography.

The blacknose sharks have highly variable single-locus $G_{S T}$ values, with the highest being 0.35 and 0.18 and the lowest being 0 and 0 for the 2- and 10-population models respectively (Figure 5C). Among the 23 microsatellites, $G_{S T}$ and $H_{S}$ estimates are moderately negatively correlated, with a correlation coefficient of $-0.41(p=0.017)$ and $-0.43(p=0.007)$ for the 2- and 10-population models respectively. None of the correlations are significant at 
$p=0.001$, but there is a clear trend of less differentiation at more polymorphic marker loci which indicates that mutations might have reduced the $G_{S T}$ values at these loci.

The differentiation calculated from each of the 10 microsatellites is low $\left(G_{S T}<0.04\right)$ among the 8 Mediterranean shore crab populations (Figure 5D). Nevertheless, $G_{S T}$ and $H_{S}$ estimates are highly negatively correlated, with a correlation coefficient of -0.80 and a small $p$ value (0.010). It is likely that mutations have substantially impacted on the $G_{S T}$ estimates from these microsatellites, and thus the underlying population differentiation due to demography may well be underestimated by these microsatellites.

The 17 microsatellites in blacktip reef sharks are highly variable in diversity, with the number of observed alleles varying from 4 to 48 and the $H_{S}$ varying from 0.15 to 0.89 . The $G_{S T}$ values among the 15 populations estimated from the 17 loci are also highly variable, from 0.04 to 0.41 (Figure 5E). The 3 loci showing deviation from Hardy-Weinberg equilibrium are apparently not outliers in terms of both diversity and differentiation. The single locus $G_{S T}$ and $H_{S}$ estimates are highly negatively correlated, with a correlation coefficient of $-0.890\left(p=0.000 \times 10^{-6}\right)$ and $-0.913\left(p=0.000 \times 10^{-6}\right)$ for the entire set of 17 loci and the subset of 14 loci respectively. In this system, mutations are highly likely to have reduced the differentiation of the microsatellites; the underlying population differentiation due to drift and migration should be higher than the average $G_{S T}$ value calculated from these microsatellites.

The differentiation measured by $G_{S T}$ at each of the 17 microsatellites is low among the 10 copper rockfish populations (Figure 5F). Except for locus Sra11-103 which has a $G_{S T}=$ 0.09 , single locus $G_{S T}$ values are below 0.05 . The overall mean $G_{S T}$ across loci is 0.027 , very close to the $F_{S T}$ value 0.031 obtained by Dick et al. (2014). Single locus $G_{S T}$ and $H_{S}$ estimates are not correlated, with a correlation coefficient of 0.011 and a $p$ value of 0.649 . It can be concluded confidently that mutations have no impact on these $G_{S T}$ estimates, and all markers, regardless of polymorphisms, should have the same expected differentiation which is equivalent to the population differentiation. The average $G_{S T}$ across loci, 0.027 , should be an unbiased estimate of the population differentiation due to demography.

\section{Discussion}

The claim that $F_{S T}$ and $G_{S T}$ are dependent on marker $H_{S}$ and underestimate population differentiation when calculated from highly polymorphic (i.e. high $H_{S}$ ) markers (e.g. 
Nagylaki 1998; Hedrick 2005; Jost 2008) can be misleading. It has led to the conclusion that these traditional statistics should be either "corrected" for $H_{S}$ (e.g. Hedrick 2005) or replaced by new statistics such as $D$ (Jost 2008). The claim creates lots of confusions, as if $F_{S T}$ and $G_{S T}$ should be independent of $H_{S}$ to be correct measures of differentiation. As Wright (1978, p.82) explicitly stated, however, $F_{S T}$ (the same for $G_{S T}$ ) measures "the amount of differentiation among subpopulations, relative to the limiting amount under complete fixation". Complete fixation means each subpopulation is fixed with an allele (i.e. all individuals in a subpopulation have the same homozygous genotype), which is not necessary to be unique among subpopulations. Fixation results in $H_{S}=0$, and the maximal differentiation of $F_{S T}=1$ is achieved only at $H_{S}=0$. For this reason, Wright (1951) also called his $F_{S T}$ a fixation index, among other fixation indices of $F_{I S}$ and $F_{I T}$. The quantity $H_{S}$ measures the absolute distance from complete fixation (i.e. $H_{S}=0$ ), and naturally constrains $F_{S T}$, which measures the relative (to total diversity $H_{T}$ ) or standardized distance from complete fixation. The definition of $G_{S T}=1-H_{S} / H_{T}$ (Nei 1973) makes the functional relationship between absolute (i.e. $H_{S}$ ) and relative (i.e. $G_{S T}$ ) differentiations explicit. Therefore, both $F_{S T}$ and $G_{S T}$ legitimately depend on, or more precisely, are constrained by $H_{S}$. This relationship is true both mathematically and biologically, and does not inherently cause $F_{S T}$ and $G_{S T}$ to underestimate differentiation for markers with high $H_{S}$.

More precisely, $F_{S T}$ and $G_{S T}$ become marker dependent and underestimate population differentiation only when migration rate is lower than mutation rate. Otherwise, they provide accurate estimates of population differentiation regardless of marker $H_{S}$. In a population with low migration rates (i.e. $m<u$ ), a marker with a higher $u$ is expected to have a higher $H_{S}$ (or absolute differentiation) and a correspondingly lower $G_{S T}$ (or relative differentiation) in both equilibrium and many non-equilibrium conditions (Whitlock 2011; this study). It should be emphasized that a high $u$ does not necessarily lead to a high $H_{S}$, and vice versa. This is because it is the quantity $u N$ rather than $u$ that determines $H_{S}$. A marker with a small $u$ in a population with a large $N$ can still harbour a high $H_{S}$, and a marker with a large $u$ in a population with a small $N$ can still have a low $H_{S}$. The statement that microsatellites, because of their high allelic polymorphisms and high $H_{S}$, must always underestimate differentiation is imprecise. Such markers show less differentiation than less polymorphic markers (e.g. SNPs) only when migration is weak $(m<u)$, as illustrated by Figures 1 and 2 .

This study reveals that whenever $m<u$ and thus mutations have a substantial impact, single locus $G_{S T}$ values decrease almost linearly with single locus $H_{S}$. This is true in both 
equilibrium (Figure 1) and non-equilibrium populations, as verified by simulations under different migration and mutation models (Figure 3). It is not surprising that the pattern observed under the ideal island migration model and the infinite allele mutation model applies to other migration and mutation models, because $G_{S T}$ and $F_{S T}$ are defined as descriptive statistics without any predefined demographic and mutation models. Mutations act to increase genetic diversity $\left(H_{S}\right.$ and $\left.H_{T}\right)$ and thus to decrease differentiation among subpopulations, no matter they occur in the finite or infinite allele model or in the stepwise mutation model (Wright 1943). Migrations, in contrast, tend to redistribute genetic diversity evenly among subpopulations. Thereby they tend to reduce the difference between $H_{S}$ and $H_{T}$ and thus to reduce $G_{S T}$, no matter they occur in the island model, stepping stone model or the isolation-by-distance model.

The simulations confirm that a correlation analysis between single locus $G_{S T}$ and $H_{S}$ estimates can be used to detect the mutational effects on differentiation. Under typical sampling intensities, the analysis has sufficient power to identify the mutational effect when it is present, and it does not falsely detect the mutational effect when it is absent (Figure 4), when the significance level is chosen as $p=0.001$. A higher significance $p$ value (say, 0.05 or $0.01)$ leads to higher powers, but also higher false detect rates. Under the finite island and infinite allele models (first row in Figure 4), for example, the power (when $m=0.001$ ) and false detection rate (when $m=0.01$ ) increase to $86.7 \%$ and $11.8 \%$ respectively when $p=0.01$, and to $90.7 \%$ and $30.0 \%$ respectively when $p=0.05$. A good balance between type I and II errors is achieved at $p=0.001$, which leads to a false detection rate being always below $7 \%$ irrespective of the widely varying sampling intensities of the number of subpopulations, the number of individuals per subpopulation, and the number of loci and polymorphisms (Figure 4).

Two out of the five empirical microsatellite datasets (Figures 5A, 5E) show strong evidence (a high negative $r_{G H}$ value and a small $p$ value) that mutations have reduced $G_{S T}$ estimated from microsatellites, two datasets (Figures 5C, 5D) indicate a similar trend with higher uncertainties, and the remaining dataset (Figure 5F) shows no detectable effect of mutations on $G_{S T}$. It is noticeable that the copper rockfish populations (Figure 5F) have high and widely variable $H_{S}$ values across the 17 microsatellites, the highest $H_{S}$ being 0.936 . These $H_{S}$ values are similar to those of the microsatellites in Atlantic salmon populations (Figure 5A) and the blacktip reef shark populations (Figure 5E). Yet, contrasting patterns of $G_{S T}$ and $H_{S}$ were observed among the three species. This again verifies the theory and simulation based 
conclusion that a high $H_{S}$ does not necessarily lead to marker dependent $G_{S T}$, and does not necessarily result in underestimation of population differentiation. In situations where the correlation between $G_{S T}$ and $H_{S}$ has a high uncertainty (e.g. Figure 5C), collection of more data (by sampling more subpopulations, loci, and individuals) may confirm or reject the hypothesis that $G_{S T}$ in a study system is affected by $H_{S}$ or mutations. In contrast, the analysis of a big SNP dataset (Figure 5B) does not detect any mutational effect. The correlation between single locus $G_{S T}$ and $H_{S}$ values, 0.044 , is small and positive, and clearly indicates no mutational effects on $G_{S T}$. The results are understandable because the $u$ for SNPs can be several orders smaller than that for microsatellites, and as a result is more likely to be smaller than migration rate $m$.

The five empirical microsatellite datasets were taken from the most recent literature at random with regard to the relationship between $G_{S T}$ and $H_{S}$, which was revealed only after the correlation analyses. If this small sample of datasets represents the reality, then we may conclude that underestimation of differentiation by microsatellites could be a common problem (Hedrick 1999, 2005; Jost 2008). A meta-analysis of many more microsatellite datasets as exemplified in this study is required for a solid conclusion. However, while microsatellites do underestimate differentiation in some (or many) situations, they can also yield unbiased estimates in situations where migration is high as shown for the copper rockfish populations (Figure 5F). The assertion that all microsatellites of high $H_{S}$ underestimate differentiation and therefore all $G_{S T}$ estimates should be standardized (Hedrick 2005) or abandoned and replaced by new differentiation statistics (Jost 2008) is unjustified. In addition to the problems shown before (Ryman \& Leimar 2009; Whitlock 2011; Wang 2012), these new statistics are also marker diversity dependent as shown below.

It is notable that several authors have conducted a meta-analysis of the relationship between $G_{S T}$ and $H_{S}$ across species/populations (Heller \& Siegismund 2009; Meirmans \& Hedrick 2011). They found that the estimated $G_{S T}$ is always smaller than the maximum value of $1-H_{S}$, as expected, and shows a weak negative correlation with $H_{S}$. It should be pointed out that the correlation analysis proposed in my study is fundamentally different from that in these meta-analyses. In the latter, the correlation is at the species level, where $G_{S T}$ and $H_{S}$ are average values across loci for each species. Because different species may have experienced different evolutionary forces and demography such that their $G_{S T}$ values differ, it is unclear what the hypothesis these meta-analyses are trying to prove or disapprove, except for the functional relationship $G_{S T}<1-H_{S}$ which should however always be true from the definition 
of $G_{S T}$. The presence of a negative correlation between $G_{S T}$ and $H_{S}$ does not prove that $G_{S T}$ is underestimated and is marker dependent because of mutational effects. The absence of the correlation does not prove that mutations have negligible effects and $G_{S T}$ is unbiased and marker independent. In my study, the correlation is between single locus values of $G_{S T}$ and $H_{S}$ within a species (population). The hypothesis, clearly defined and supported by theory and simulations, is that $G_{S T}$ values should be similar across markers of different $H_{S}$ if mutations are unimportant (when $u<m$ ), resulting in an $r_{G H}$ not different from 0. Otherwise (i.e. $u>m$ ), $G_{S T}$ values should decrease with markers showing an increasing $H_{S}$, resulting in a highly negative correlation between $G_{S T}$ and $H_{S}$.

$G_{S T}$ calculated from a locus measures the genetic differentiation among subpopulations at the locus due to the combined effect of all evolutionary forces (Nei 1973). Selection directly influences $F_{S T}$ and $G_{S T}$, as Wright (1943) illustrated with several different types of selection. In principle, a negative correlation between $H_{S}$ and $G_{S T}$ can also be generated for markers closely linked with a locus under strong selection for spatially different alleles (which causes a decrease in $H_{S}$ and an increase in $G_{S T}$ ) or/and for spatially different allele combinations (which causes an increase in $H_{S}$ and a decrease in $G_{S T}$ ). Although my correlation analysis assumes the absence of selection, it should be robust in most applications. First, frequently only a few microsatellites $(<30)$ are used in calculating $F_{S T}$ or $G_{S T}$, and the chance of any of them being under selection or being linked to loci under selection strong enough (compared with other evolutionary forces) for detection is slim. Second, with genomic dense markers such as SNPs, it is highly likely that a small fraction of the loci are under strong selection. The correlation analysis should however still be robust because the vast majority of loci are neutral and a few selected loci should not affect the overall relationship between $H_{S}$ and $G_{S T}$.

This study focusses on the widely applied differentiation statistic $G_{S T}$ (Nei 1973). Other differentiation statistics or estimators such as $\theta$ (Weir \& Cockerham 1984), $D$ (Jost 2008 ) and $G_{S T}^{\prime}$ (Hedrick 2005) could also be affected by mutations and yield marker $\left(H_{S}\right)$ dependent estimates. All these statistics measure differentiation at marker loci due to the collective actions of all evolutionary forces, including mutations. When mutations are important (i.e. $u>m$ ), therefore, differentiation estimates are expected to be different among loci. Some statistics, like $D$ which is claimed to outperform $G_{S T}$ for highly polymorphic markers (Jost 2008), are even more problematic and produce marker dependent differentiation estimates even when mutation rate is small relative to migration rate. For the 
data simulated in finite island and infinite allele models, finite island and stepwise mutation models, and stepping stone and stepwise mutation models shown in Figure 3, for example, the correlation coefficient between $D$ and $H_{S}, r_{D H}$, is $0.43,0.30$, and 0.22 respectively when $m=0.001$, and is $0.71,0.24$ and 0.26 respectively when $m=0.01$. The correlation is always positive and substantially high, even in the situation where mutation is very weak relative to migration and $G_{S T}$ is uncorrelated with $H_{S}$. Similarly highly positive $r_{D H}$ values are obtained for all of the empirical datasets. For the Atlantic salmon SNP dataset, $r_{D H}$ is 0.73 while $r_{G H}$ is only 0.04 . This means $D$ always increases with $H_{S}$, even for markers with low mutation rate (e.g. SNPs) and low diversity, and for a population with a high migration rate.

Slatkin's (1995) $R_{S T}$ provides unbiased estimates of population differentiation regardless of the mutation rates or diversity of markers. A mutation does not erase the evolutionary history of a gene when it occurs in some models such as the stepwise model. Mutations occurring in these models are accommodated by $R_{S T}$, which therefore measures differentiation purely due to population demography ( $m$ and $N$ ). Unfortunately, however, $R_{S T}$ is sensitive to violations of the assumed mutation models and have a higher sampling variance than $G_{S T}$ (Balloux \& Lugon-Moulin 2002). Unless many (say in the hundreds) markers are used, $R_{S T}$ may have a lower accuracy than $G_{S T}$.

What are the uses of a correlation analysis on $G_{S T}$ and $H_{S}$ ? What we are usually interested are population level forces such as migration (or isolation) and drift, which have roughly the same effect on all loci in the genome, and population differentiation, which depends on population level forces and is estimated by all loci mainly controlled by population level forces. $G_{S T}$ always faithfully reflects the differentiation at the marker loci, no matter the loci are governed primarily by population demography ( $m$ and $N$ ) or locus specific forces such as selection and mutation. Marker $G_{S T}$ provides an unbiased and good estimate of population differentiation only when these markers are not significantly affected by locus specific forces. The correlation analysis essentially tests whether different markers give replicated or different estimates of $G_{S T}$, or whether or not population level forces are much more important than locus specific forces in shaping the marker diversity and distribution. A highly negative correlation between $G_{S T}$ and $H_{S}$ values indicates that 1) the migration rate must be low, lower than the mutation rate; 2) the marker $G_{S T}$ may well underestimate population differentiation; 3) another set of markers with lower (higher) polymorphisms may well yield a higher (lower) estimate of $G_{S T} ; 4$ ) the marker $G_{S T}$ should be used cautiously in comparisons across species, studies and sets of loci. If the correlation between $G_{S T}$ and $H_{S}$ 
560

561

562

563

564

565

566

567

568

569

570

571

572

573

574

575

576

577

578

579

580

581

582

583

584

585

586

587

588

589

590

values among loci is small and non-significant, then these single locus $G_{S T}$ estimates should be marker (diversity) independent and can be averaged to provide a good estimate of population differentiation.

A computer program, CoDiDi (Correlation between Diversity and Diferentiation), is written to calculate single locus $G_{S T}$ and $H_{S}$ values, to test whether a single locus $G_{S T}$ value is significantly different from 0 or not by permutations, and to calculate and test the significance of the correlation between $G_{S T}$ and $H_{S}$. The correlation analyses of all of the simulated and empirical data presented in this study were conducted by this program, freely available from the website: http://www.zsl.org/science/software/CoDiDi.

\section{Acknowledgments}

I thank PW Hedrick, PG Meirmans, N Ryman, Robin Waples, MC Whitlock and an anonymous referee for valuable comments on earlier versions of this manuscript.

\section{References}

Balloux F, Lugon-Moulin N (2002) The estimation of population differentiation with microsatellite markers. Molecular Ecology, 11, 155-165.

Balloux F, Brunner H, Lugon-Moulin N, Hausser J, Goudet J (2000) Microsatellites can be misleading: an empirical and simulation study. Evolution, 54, 1414-1422.

Carreras-Carbonell J, Macpherson E, Pascual M (2006) Population structure within and between subspecies of the Mediterranean triplefin fish Tripterygion delaisi revealed by highly polymorphic microsatellite loci. Molecular Ecology, 15, 3527-3539.

Dick S, Shurin JB, Taylor EB (2014) Replicate divergence between and within sounds in a marine fish: the copper rockfish (Sebastes caurinus). Molecular Ecology, 23, 575-590.

Hedrick PW (1999) Perspective: highly variable loci and their interpretation in evolution and conservation. Evolution, 53, 313-318.

Hedrick PW (2005). A standardized genetic differentiation measure. Evolution, 59, 16331638.

Heller R, Siegismund H (2009) Relationship between three measures of genetic differentiation $\mathrm{G}_{\mathrm{ST}}$, D $\mathrm{EST}$ and $G$ 'st : how wrong have we been? Molecular Ecology, 18, 2080-2083. 
591

592

593

594

595

596

597

598

599

600

601

602

603

604

605

606

607

608

609

610

611

612

613

614

615

616

617

618

619

620

621

622

623

Jin L, Chakraborty R (1995) Population structure, stepwise mutation, heterozygote deficiency and their implications in DNA forensics. Heredity, 74, 274-285.

Jost L (2008) $G_{S T}$ and its relatives do not measure differentiation. Molecular Ecology, 17, 4015-4026.

Kimura M, Crow JF (1964) The number of alleles that can be maintained in a finite population. Genetics, 49, 725-738.

Kimura M, Weiss G (1964) The stepping-stone model of population structure and the decrease of genetic correlation with distance. Genetics, 49, 561-576.

Li W-H (1976) Effect of migration on genetic distance. American Naturalist, 110, 841-847.

Meirmans PG, Hedrick PW (2011) Assessing population structure: $F_{S T}$ and related measures. Molecular Ecology Resources, 11, 5-18.

Moore JS, Bourret V, Dionne M et al. (2014) Conservation genomics of anadromous Atlantic salmon across its North American range: outlier loci identify the same patterns of population structure as neutral loci. Molecular Ecology, 23, 5680-5697.

Nagylaki T (1998) Fixation indices in subdivided populations. Genetics, 148, 1325-1332.

Nei M (1973) Analysis of gene diversity in subdivided populations. Proceedings of the National Academy of Sciences of the United States of America, 70, 3321-3323.

Nei M (1975) Molecular Population Genetics and Evolution. North-Holland, Amsterdam, Netherlands.

Nei M (1977) F-statistics and analysis of gene diversity in subdivided populations. Annals of Human Genetics, 41, 225-233.

Nei M, Chesser R (1983) Estimation of fixation indices and gene diversities. Annals of Human Genetics, 47, 253-259.

Ohta T, Kimura M (1973) A model of mutation appropriate to estimate the number of electrophoretically detectable alleles in a finite population. Genetical Research, 22, 201204.

Portnoy DS, Hollenbeck CM, Belcher CN et al. (2014) Contemporary population structure and post-glacial genetic demography in a migratory marine species, the blacknose shark, Carcharhinus acronotus. Molecular Ecology, 23, 5480-5495.

Pritchard JK, Stephens M, Donnelly P (2000) Inference of population structure using multilocus genotype data. Genetics, 155, 945-959.

Ryman N, Leimar O (2008) Effect of mutation in genetic differentiation among nonequilibrium populations. Evolution, 62, 2250-2259. 
Ryman N, Leimar O (2009) $G_{S T}$ is still a useful measure of genetic differentiation-a comment on Jost's D. Molecular Ecology, 18, 2084-2087.

Sanetra M, Crozier R (2003) Patterns of population subdivision and gene flow in the ant Nothomyrmecia macrops reflected in microsatellite and mitochondrial DNA markers. Molecular Ecology, 12, 2281-2295.

Schiavina M, Marino IAM, Zane L, Melià P (2014) Matching oceanography and genetics at the basin scale. Seascape connectivity of the Mediterranean shore crab in the Adriatic Sea. Molecular Ecology, 23, 5496-5507.

Slatkin M (1995) A measure of population subdivision based on microsatellite allele frequencies. Genetics, 139, 457-462.

Takahata N, Nei M (1984) $F_{S T}$ and $G_{S T}$ statistics in the finite island model. Genetics, 107, 501-504.

Vignaud TM, Mourier J, Maynard JA et al. (2014). Blacktip reef sharks, Carcharhinus melanopterus, have high genetic structure and varying demographic histories in their Indo-Pacific range. Molecular Ecology, 23, 5193-5207.

Wang J (2012) On the measurements of genetic differentiation among populations. Genetics Research, 94, 275-289.

Wang J, Caballero A (1999) Developments in predicting the effective size of subdivided populations. Heredity, 82, 212-226.

Weir BS, Cockerham CC (1984) Estimating $F$-statistics for the analysis of population structure. Evolution, 38, 1358-1370.

Whitlock MC (2011) $G_{S T}$ and $D$ do not replace $F_{S T}$. Molecular Ecology, 20, 1083-1091.

Wright S (1931) Evolution in Mendelian populations. Genetics, 16, 97-159.

Wright S (1943) Isolation by distance. Genetics, 28, 114-138.

Wright S (1951) The genetical structure of populations. Annals of Eugenics, 15, 323-354.

Wright S (1969) Evolution and the Genetics of Populations, Vol. II. The Theory of Gene Frequencies. University of Chicago Press, Chicago.

Wright S (1978) Evolution and the Genetics of Populations, Vol. IV. Variability Within and Among Natural Populations. University of Chicago Press, Chicago. 
$655 \mathrm{~J}$. Wang is interested in developing population genetics models and methods of analysis of

656

657

658

\section{Data accessibility}

660 The computer program for simulating genotype data under different migration and mutation

661 models, and for estimating $H_{S}, G_{S T}$ and their correlation: Dryad DOI: 10.5061/dryad.733s9.

662 The 6 empirical datasets were retrieved from Dryad with DOIs:

663 http://dx.doi.org/10.5061/dryad.sb601; http://dx.doi.org/10.5061/dryad.vv277;

664 http://dx.doi.org/10.5061/dryad.r0d1q; http://dx.doi.org/10.5061/dryad.th4h5;

665 http://dx.doi.org/10.5061/dryad.s489b

666 The input files of the 6 empirical datasets for CoDiDi analysis: Dryad DOI:

$667 \quad 10.5061 /$ dryad.733s 9. 
Table 1 Parameter ranges in simulations

\begin{tabular}{|c|c|c|c|c|c|c|c|}
\hline $\begin{array}{c}\text { Migration } \\
\text { model }\end{array}$ & $\begin{array}{c}\text { Mutation } \\
\text { Model }\end{array}$ & $t$ & $m$ & $u$ & $M$ & $R$ & $L$ \\
\hline FIM, & IAM, & $200, \propto$ & $0.01,0.001$ & $10^{-5} \sim 10^{-3}$ & $10,20,40$, & $2,3,4,6$, & 5,10, \\
SSM & SWM, & & & & 80,160 & $8,10,12$ & 15,20, \\
& FAM & & & & & & 30 \\
\hline
\end{tabular}

670 The size $(N)$ and number (s) of subpopulations are fixed at 250 (or 1000) and 20, respectively.

671 The finite island model (FIM) and circular stepping stone model (SSM) for migrations are

672 considered for neutral loci under infinite allele model (IAM), stepwise model (SWM) or

673 finite allele model (FAM) for mutations. For FAM, 2 alleles are considered to mimic SNPs.

674 Symbols $t, m, u, M, R, L$ represent number of generations when sampling occurs, migration

675 rate, mutation rate, number of individuals sampled from a subpopulation, number of sampled

676 subpopulations, and number of sampled loci, where $t=\propto$ indicates a population at mutation-

677 drift-migration equilibrium. 


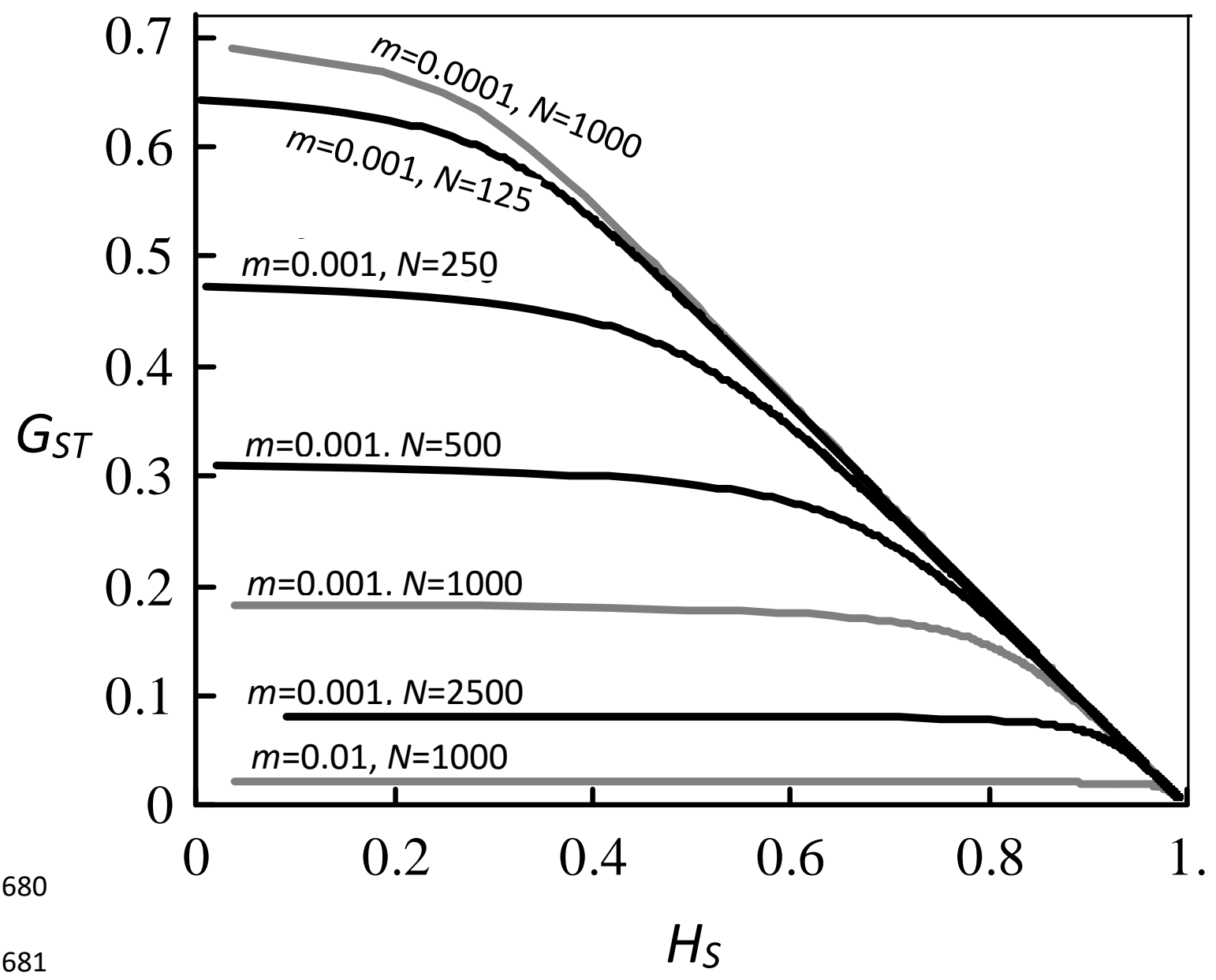

682

683

684

685

686

687

688 Fig. $1 G_{S T}$ as a function of $H_{S}$ in equilibrium populations. The $G_{S T}\left(y\right.$ axis) and $H_{S}$ ( $x$ axis)

689 values for a population in a finite island model with $s=10$ subpopulations at mutation-drift-

690 migration equilibrium were calculated for various parameter values of subpopulation size $(N)$,

691 migration rate $(m)$, and mutation rate $(u)$, where $u$ ranges from $10^{-6}$ (left side of $x$ axis) to $10^{-2}$ 692 (right side of $x$ axis). 
694

695

696

697

698

699

700

701

702

703

704

705

706

707
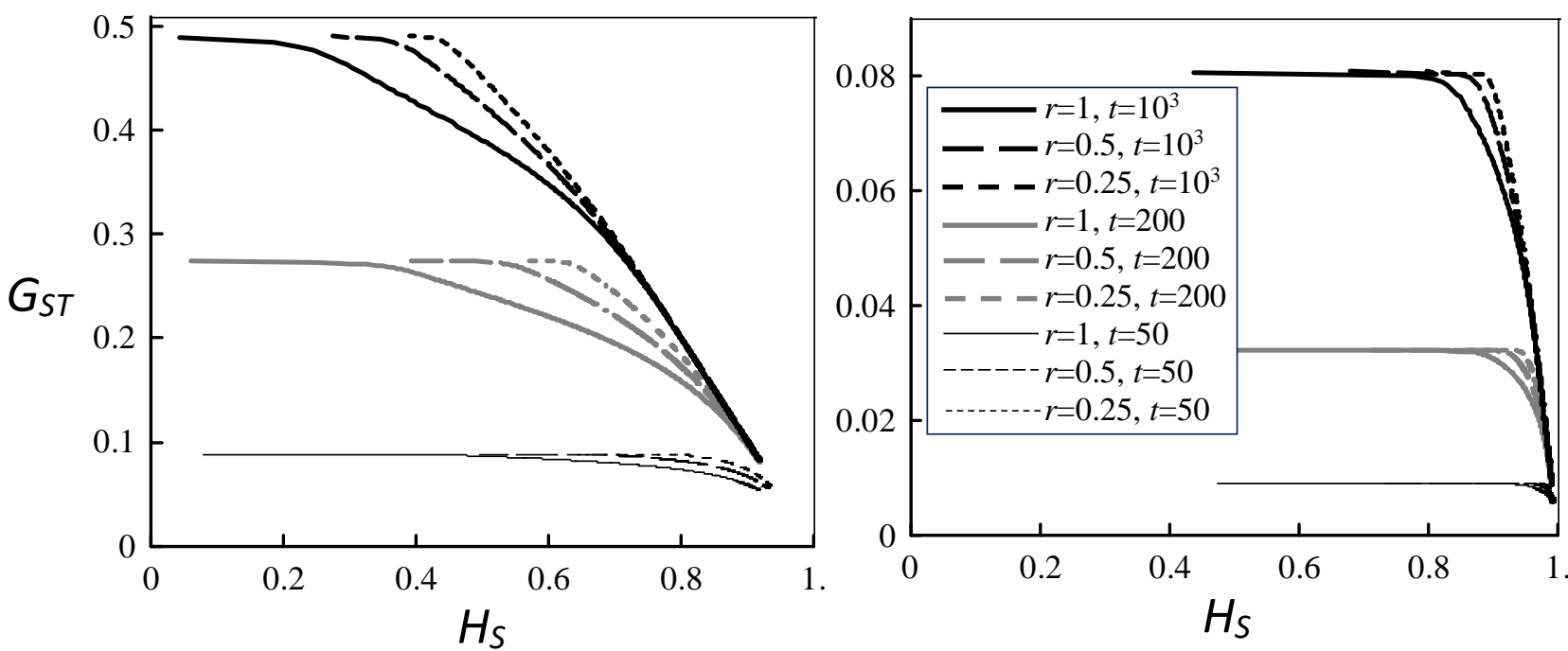

65

\section{6}

7

8

1

Fig. $2 G_{S T}$ as a function of $H_{S}$ in non-equilibrium populations. The $G_{S T}$ ( $y$ axis) values are plotted against $H_{S}$ ( $x$ axis) values at different generations $(t=50,200,1000)$ for a population in a finite island model with $s=10$ subpopulations, assuming parameter values of $N=250$ (left panel) or 1000 (right panel), $m=0.001$, and a variable $u$ ranging from $10^{-6}$ (left side of $x$ axis) to $10^{-2}$ (right side of $x$ axis). The initial probability of gene identity is assumed to be $r J_{0(\propto)}$, . where $r=1,0.5$ and 0.25 and $J_{0(\infty)}$ is the equilibrium value of $J_{0}$ given parameters $N, m, u, s$. 

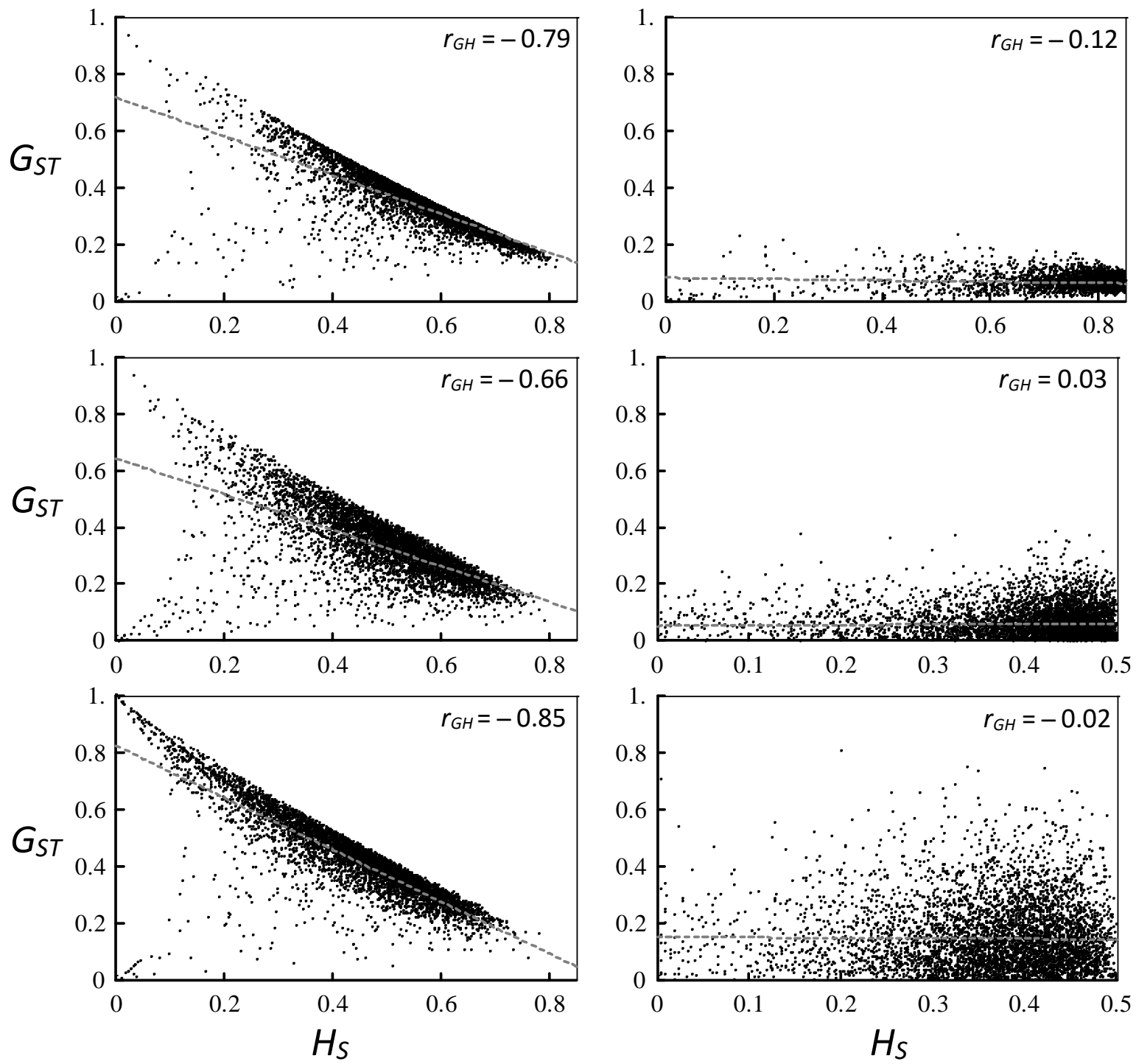

Fig. 3 Scatter graphs of $G_{S T}\left(y\right.$ axis) and $H_{S}$ ( $x$ axis) estimates in mutation-drift-migration equilibrium populations. The population parameters are $N=250, s=20, u$ is taken at random from a uniform distribution in the range $\left[10^{-5}, 10^{-3}\right]$, and migration rate is either $m=0.001$ (left column) or $m=0.01$ (right column). The population is assumed to follow the finite island and infinite allele models (first row), finite island and stepwise mutation models (second row), or stepping stone and stepwise mutation models (third row). For each graph, 5000 replicate simulated datasets (loci) were generated to estimate $G_{S T}$ and $H_{S}$, using $R=4$ (out of $s=20$ ) randomly sampled subpopulations and $M=50$ (out of $N=250$ or 1000) randomly sampled individuals per subpopulation. The correlation between the $G_{S T}$ and $H_{S}$ estimates for each graph is shown at the right corner of the graph. 
723

724

725

726

727

728

729

730

731

732

733

734

735

736

737
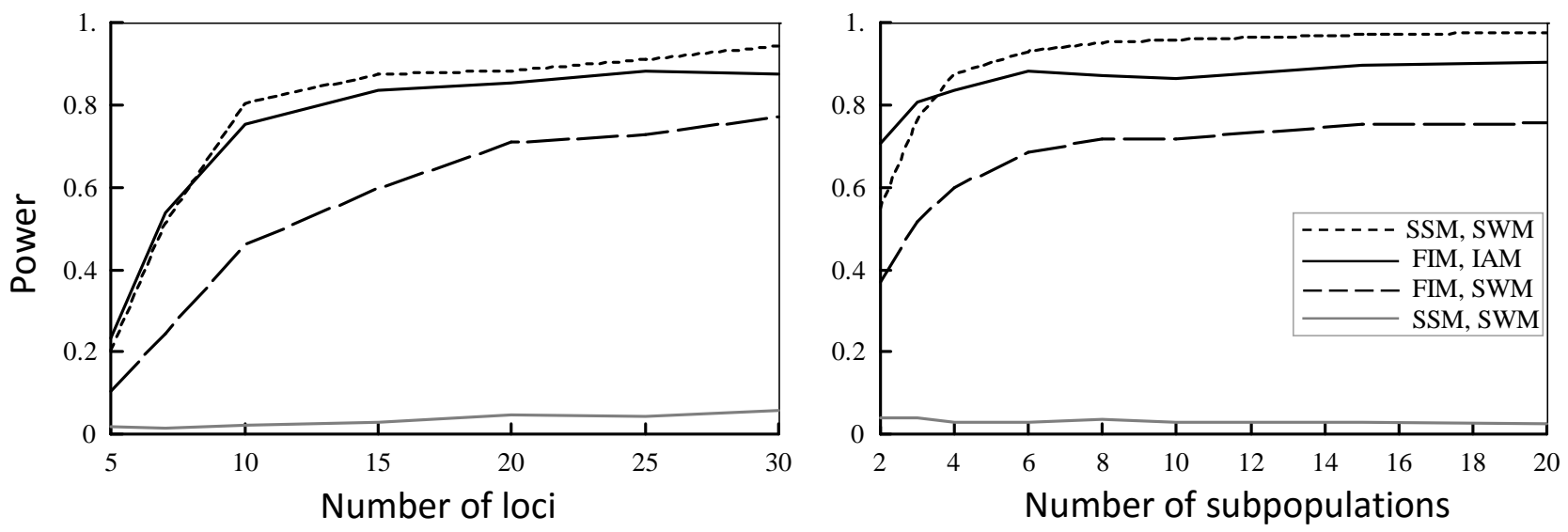

Fig. 4 Power of correlation analysis of $G_{S T}$ and $H_{S}$ estimates in mutation-drift-migration equilibrium populations. The population parameters are $N=250, s=20$, and $u$ is taken at random from a uniform distribution in the range $\left[10^{-5}, 10^{-3}\right]$. The numbers of sampled subpopulations and loci are 4 and variable for the left panel, or variable and 15 for the right panel. Migration rate is either $m=0.001$ (black continuous, black broken and black dotted lines) or $m=0.01$ (grey continuous lines). The population is assumed to follow the finite island model (FIM) and infinite allele model (IAM), finite island model and stepwise mutation model (SWM), or stepping stone model (SSM) and stepwise mutation model. For each parameter combination, the proportion of 1000 replicate datasets in which the correlation coefficient between $G_{S T}$ and $H_{S}$, estimated using 40 individuals per sampled subpopulation, is statistically significant at $p<0.001$ is plotted (on $y$ axis) as a function of the number of sampled loci (left panel) or the number of sampled subpopulations (right panel) (on $x$ axis). The black lines show the power in detecting mutational effects on $G_{S T}$ when such effects exist (i.e. when migrations are weak relative to mutations, $m=0.001$ ), and the grey lines show the false detection rates when mutational effects are absent (i.e. when migrations are strong relative to mutations, $m=0.01$ ). 

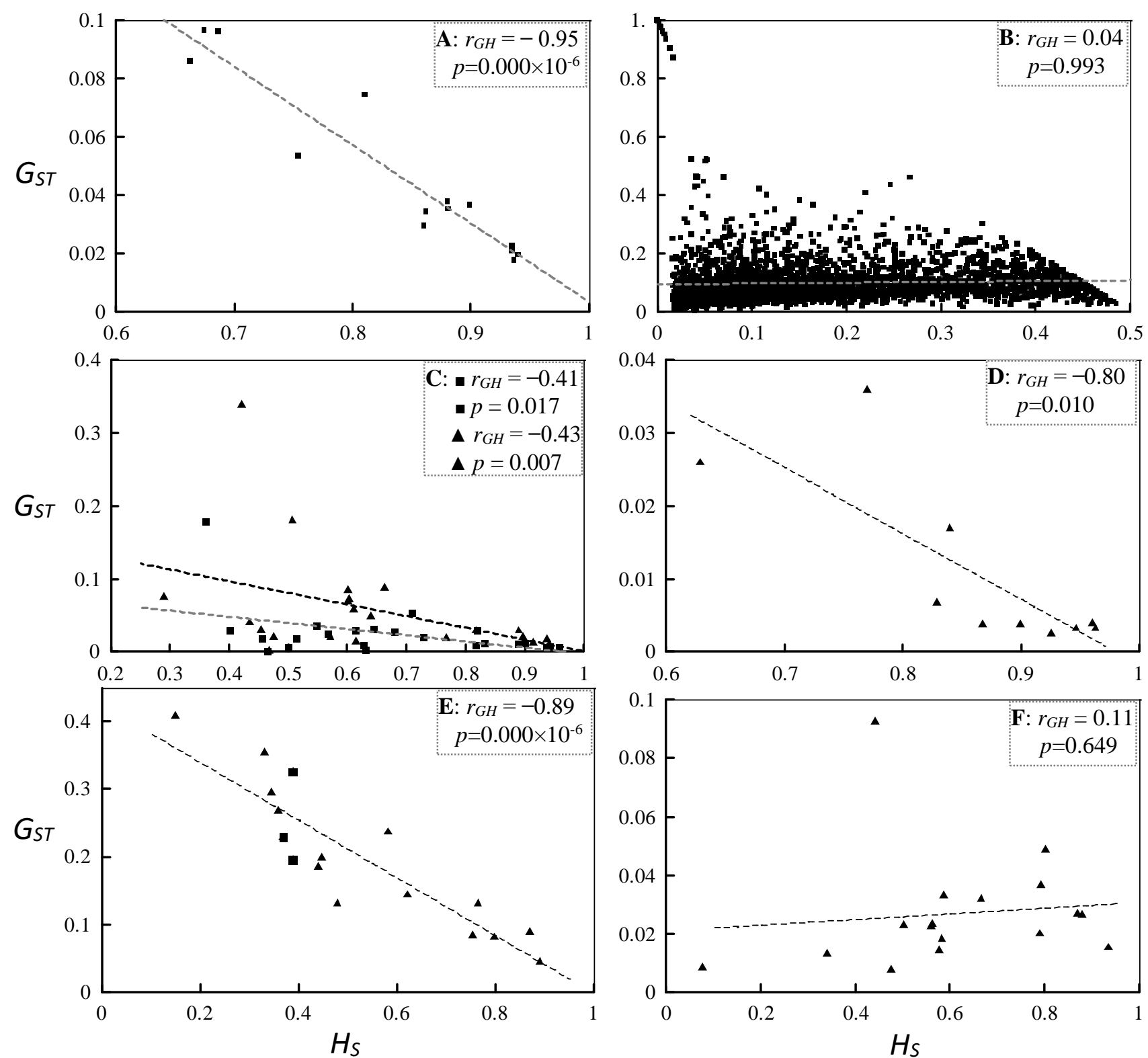

753 Fig. 5 The relationship between single locus $G_{S T}$ and $H_{S}$ estimates in empirical datasets. The 754 correlation coefficient between $G_{S T}$ and $H_{S}$ and the $p$ value for each dataset are shown at the 755 top right corner of each graph, and the grey dotted lines show the fitted regression of $G_{S T}$ on 
$756 H_{S}$. Graphs A and B show the results for the 15 microsatellites and 3129 SNPs respectively in

757 North American Atlantic salmon populations. Graph C shows the results for the 23

758 microsatellites in the blacknose shark populations, where each triangle and each square

759 shows the pair of $G_{S T}$ and $H_{S}$ values estimated from a single marker in the 2- and 10-

760 population models, respectively. Graph D shows the results for the 10 microsatellites in eight

761 Mediterranean shore crab populations. Graph E shows the results for the 17 microsatellites in

76215 blacktip reef shark populations, where each triangle and each square represents a single

763 marker without and with deviation from Hardy-Weinberg equilibrium. Graph F shows the

764 results for the 17 microsatellites in 10 copper rockfish populations. 